\title{
Analyse des pratiques de détection des chaleurs dans les élevages bovins laitiers algériens
}

\author{
A. Yahimi ${ }^{1 *}$ N. Djellata ${ }^{1}$ C. Hanzen ${ }^{2}$ R. Kaidi ${ }^{1,3}$
}

\author{
Mots-clés \\ Bovin - Vache laitière - Détection de \\ I'œstrus - Reproduction - Algérie.
}

\begin{abstract}
Résumé
Une enquête sur les pratiques de détection des chaleurs a été réalisée dans 222 exploitations bovines, réparties dans cinq régions (wilayas) du centre de l'Algérie (Tizi Ouzou, Blida, Médéa, Tipaza et Bouira). Le questionnaire utilisé contenait des informations générales relatives à l'élevage (nombre de femelles, stabulation, spécialisation) mais aussi les pratiques de détection des chaleurs (périodes d'observation, signes observés) et les moyens complémentaires éventuellement utilisés. La majorité des éleveurs (57 p. 100) ont été confrontés à un problème de détection des chaleurs. Dans 49 p. 100 des cas seulement, ils inséminaient pour la première fois leurs vaches 50 à 90 jours après le vêlage. Un éleveur sur deux (53 p. 100) détectait les chaleurs deux fois par jour et 40 p. 100 les observaient trois fois par jour, cette activité étant pratiquée de manière aléatoire. Ils basaient prioritairement leur constat d'œstrus sur la présence d'un écoulement vulvaire (19 p. 100) ou sur l'acceptation du chevauchement (16 p. 100). La taille du troupeau ou la spécialisation ont été sans influence sur la fréquence des signes considérés. Les vaches n'ayant pas présenté des signes de chaleurs au cours des 60 premiers jours suivant le vêlage ont fait l'objet d'un examen clinique par un vétérinaire dans 76 p. 100 des élevages. Les moyens complémentaires de détection tels que le calendrier rotatif, ou le crayon détecteur n'ont été utilisés que par 34 p. 100 des éleveurs. Les facteurs responsables de la manifestation des chaleurs, les moments et le nombre d'observations, I'examen des anœstrus post-partum et I'utilisation des moyens complémentaires ont été influencés par la spécialisation et la taille de l'élevage, contrairement aux signes des chaleurs, la période d'attente et la difficulté de détection.
\end{abstract}

Le secteur laitier constitue un domaine stratégique dans le développement économique et social de l'Algérie. L'élevage bovin laitier assure actuellement 40 p. 100 de la production laitière nationale, le reste étant assuré par les importations de poudre de lait. Selon le ministère de l'Agriculture et du Développement rural (2007) (21), le cheptel bovin laitier est estimé à 900000 têtes de races autochtones et étrangères importées (Fleckvieh, Holstein Montbéliard et Brune des Alpes) produisant en moyenne entre 6 et 15 litres de lait par jour (10). On estime à 13775 le nombre de

\footnotetext{
1. Département vétérinaire, faculté des Sciences agrovétérinaires, Université Saad Dahleb, BP 270, Ouled Yaïch, 09000 Blida, Algérie.

2. Service de thériogénologie des animaux de production, faculté de Médecine vétérinaire, Université de Liège, Liège, Belgique.

3. Laboratoire de recherche des biotechnologies liées à la reproduction animale, Université Saad Dahleb, Blida, Algérie.

* Auteur pour la correspondance

E-mail : yahimi72@yahoo.fr
}

vaches importées en 2009 (22). Le déficit de production laitière est donc particulièrement important. Il est imputable à divers facteurs parmi lesquels on peut raisonnablement citer l'infécondité, le manque d'une politique rigoureuse de sélection génétique, l'inadéquation entre les besoins alimentaires et les apports disponibles, et un mauvais état sanitaire de la mamelle. Quelques publications ont précisé les performances de reproduction du cheptel laitier algérien. Elles ne concernent malheureusement qu'un nombre relativement restreint d'animaux compris entre 38 et 99 vaches. Elles font état d'un intervalle moyen entre vêlages compris entre 444 et 470 jours, et d'un intervalle moyen entre vêlages et insémination fécondante compris entre 160 et 193 jours (2). La détection des chaleurs conditionne l'obtention d'un intervalle entre le vêlage et la première insémination (période d'attente) dont la valeur moyenne au niveau du troupeau ne doit pas être supérieure à 60 jours (28). Elle influence, par ailleurs, directement la fertilité et donc la durée de la période de reproduction, puisque normalement l'insémination artificielle doit être réalisée une douzaine d'heures après le début de l'œstrus (6). Enfin, l'absence de détection du retour des chaleurs de l'animal constitue une des méthodes 
précoces de diagnostic de gravidité. La qualité de la détection des chaleurs dépend de facteurs liés à l'éleveur, et à l'animal et à son environnement. Divers facteurs liés à l'animal sont de nature à influencer l'expression du comportement ostral. Ce dernier est peu héritable $(0,21$; réf. 31$)$. D'après plusieurs auteurs, les boiteries sont classiquement associées à une diminution de l'intensité de l'œstrus chez la vache (5). De même, une mauvaise alimentation ou un bilan énergétique négatif peut affecter négativement l'expression de l'œstrus $(8,20,29)$. Les conditions environnementales de l'élevage sont également de nature à influencer la qualité de la détection des chaleurs ainsi que l'activité sexuelle des animaux $(23,27)$. Au nombre de celles-ci on peut citer la saison (9), la longueur des jours (24) et le type de stabulation (17). Orihuela (23) rapporte que l'intensité des chaleurs chez les vaches est réduite en fin d'automne et au début de l'hiver par rapport à la période estivale. De même une hygrométrie élevée contribue à diminuer l'expression des chaleurs $(14,23)$. Divers auteurs $(28,34)$ rapportent également que le caractère glissant des sols de la stabulation constitue un facteur limitant la bonne expression des chaleurs.

La présence d'un taureau est de nature à réduire la durée de la période d'anœstrus du post-partum $(12,23)$. Des études ont montré que l'activité sexuelle s'intensifiait avec le nombre des animaux en chaleur en même temps (13). Selon Hurnik et coll. (14), le nombre de montes en moyenne par vache est de 11 pour une seule vache en chaleur, ce nombre passera respectivement à 36 et 56 en cas de deux et trois vaches en chaleur simultanément. Pour Kilgour et coll. (15), lorsque l'œstrus est synchronisé dans un troupeau, une proportion plus élevée des animaux participe à la formation du groupe sexuellement actif en raison d'une stimulation sexuelle. Les moyens complémentaires, tels que les détecteurs électroniques de chevauchement $(1,30)$, les podomètres $(25,29)$, les systèmes de mesure de l'impédance électrique du mucus vaginal (28) et les animaux détecteurs équipés de systèmes de marquage des animaux en chaleur (37), constituent des méthodes qui contribuent à améliorer la qualité de la détection. La présente étude a eu pour but de caractériser le contexte et les pratiques de détection des chaleurs dans les élevages laitiers algériens et d'en analyser les facteurs potentiels d'influence.

\section{- MATERIEL ET METHODES}

L'enquête a concerné 222 exploitations laitières de cinq régions (wilayas) (Blida, Tizi Ouzou, Médéa, Tipaza et Bouira). Ces régions sont considérées comme les plus importantes pour la production laitière en Algérie. Elles comprennent respectivement 626, $640,160,380$ et 612 éleveurs laitiers qui possèdent en moyenne 45 bovins (6 à 150). Chaque éleveur a fait l'objet d'un entretien personnel pour répondre à un questionnaire divisé en deux parties. La première a concerné des questions d'ordre général, telles que le nombre de femelles à la reproduction (respectivement 10 à 19, 20 à 60 et plus de 60 femelles âgées de plus de 12 mois), la spécialisation (laitière, ou mixte laitière et à viande), le type de stabulation (libre ou entravée), les difficultés éprouvées par l'éleveur à détecter les chaleurs et leurs causes (d'après l'éleveur), la durée de la période d'attente souhaitée, la présence ou non d'un suivi vétérinaire (l'examen régulier des vaches en phase d'involution utérine ou en anœstrus du post-partum ainsi que les constats de gravidité). La deuxième partie a concerné les pratiques d'observation adoptées par l'éleveur : notation ou pas de date des chaleurs, nombre, moment et durée des périodes d'observation, signes utilisés pour le diagnostic d'œstrus, examen clinique par un vétérinaire appelé en consultation des seuls animaux en anœstrus du post-partum, moyens complémentaires éventuellement utilisés (taureau détecteur, crayon marqueur, calendrier rotatif).
Les données ainsi collectées ont fait l'objet d'une étude descriptive et relationnelle. Dans l'étude descriptive, les taux des paramètres étudiés ont été précisés. Dans l'étude relationnelle, l'influence des divers paramètres généraux ou spécifiques a été analysée statistiquement. L'identification de l'impact de la taille du troupeau et du type de spécialisation sur les paramètres étudiés a été réalisée à l'aide de logiciel R, version 2.10.1, en utilisant le test exact de Fisher.

\section{RESULTATS}

\section{Contexte de l'enquête}

La population d'étude (222 exploitations) était composée de 37 p. 100 d'exploitations mixtes et 63 p. 100 d'exploitations laitières. Ces exploitations comportaient respectivement dans 39, 37 et 24 p. 100 des cas, 10 à 19, 20 à 60, et plus de 60 femelles en âge de reproduction. Seules 42 p. 100 des exploitations faisaient l'objet d'un suivi régulier par un vétérinaire. Ce suivi était significativement $(\mathrm{p}<0,01)$ plus souvent mis en place dans les exploitations de petite taille (10 à 19 femelles) que de grande taille ( $>60$ femelles). Il était par ailleurs significativement $(\mathrm{p}<0,05)$ plus souvent appliqué dans les exploitations laitières que mixtes. Au total, 39 p. 100 des éleveurs considéraient que leurs vaches et génisses manifestaient clairement les chaleurs. Cet avis s'exprimait davantage $(\mathrm{p}<0,001)$ dans les élevages de petite taille que dans ceux de grande taille : 79 vs 18 p. 100) pour les élevages comportant respectivement moins de 20 ou plus de 60 vaches. En revanche, le type de spécialisation des élevages était sans influence sur la qualité des manifestations œstrales constatées par les éleveurs.

Une majorité d'éleveurs (57 p. 100) ont admis avoir des difficultés à détecter les chaleurs. Cette difficulté était indépendante de la taille de l'exploitation. La cause selon eux aurait été liée à l'alimentation (38 p. 100), au manque de temps pour l'observation (22 p. 100), à la race (18 p. 100), à la stabulation entravée $(13$ p. 100$)$, à la production laitière $(8$ p. 100), et génétique (2 p. 100). Les raisons évoquées par les éleveurs n'étaient pas significativement influencées par la taille de l'élevage. Cependant, l'alimentation, le manque de temps pour l'observation et la production laitière étaient davantage évoqués dans les élevages de plus de 60 femelles. Inversement, le facteur le plus souvent identifié dans les élevages de petite taille était la race.

Les raisons évoquées par les éleveurs pour expliquer leur difficulté à détecter les chaleurs étaient significativement différentes selon les spécialisations. Ainsi, dans les exploitations de type mixte la cause était alimentaire, tandis que la race et le type de stabulation étaient davantage mis en avant dans les exploitations de type laitier (tableau I).

\section{Méthodes de détection des chaleurs}

Une faible majorité d'éleveurs (53 p. 100) ne faisaient que deux périodes d'observation journalière des chaleurs et 39 p. 100 en faisaient trois. Le nombre d'observations a augmenté significativement $(p<0,001)$ avec la taille du troupeau (tableau II). En revanche, il n'a pas différé en fonction de la spécialisation de l'élevage $(p>0,05)$. Les éleveurs basaient leur diagnostic d'œstrus, par ordre d'importance, sur l'identification d'un écoulement vulvaire (19 p. 100), l'acceptation du chevauchement (16 p. 100), la nervosité (14 p. 100), la chute de la production laitière (12 p. 100), l'écoulement vulvaire muco-sanguinolant (11 p. 100), la monte active par l'avant ( 9 p. 100), la monte active par l'arrière (8 p. 100), le reniflement vulvaire (7 p. 100), le mouvement de Flehmen (4 p. 100), la pose du menton sur l'encolure ou le bassin 


\section{Tableau I}

Effet de la spécialisation sur la répartition en pourcentage des facteurs responsables $d^{\prime}$ un manque de manifestation des chaleurs $(p<0,01)$ $d^{\prime}$ après les éleveurs

$\begin{array}{lrr}\text { Facteur } & \text { Laitière } & \text { Mixte } \\ \text { Alimentation } & 30 & 50 \\ \text { Manque de temps d'observation } & 22 & 22 \\ \text { Génétique } & 2 & 0 \\ \text { Production laitière } & 5 & 14 \\ \text { Race } & 25 & 6 \\ \text { Stabulation } & 16 & 8 \\ \text { Total } & 100 & 100\end{array}$

\section{Tableau II}

Effets de la taille du troupeau sur la répartition en pourcentage des modalités pratiques de détection

\begin{tabular}{|c|c|c|c|c|}
\hline $\begin{array}{l}\text { Nb. d'animaux } \\
\text { dans le troupeau }\end{array}$ & 10 à 19 & 20 à 60 & $>60$ & Test \\
\hline Moment d'observation & & & & $P<0,001$ \\
\hline $\begin{array}{l}\text { Pendant la distribution } \\
\text { d'aliment }\end{array}$ & 33 & 30 & 60 & \\
\hline Avant la traite & 22 & 20 & 17 & \\
\hline Moment non spécifique & 45 & 50 & 23 & \\
\hline Total & 100 & 100 & 100 & \\
\hline $\begin{array}{l}\text { Nb. d'observations } \\
\text { journalières }\end{array}$ & & & & $P<0,001$ \\
\hline Une fois & 12 & 7 & 3 & \\
\hline Deux fois & 54 & 70 & 25 & \\
\hline Plus de trois fois & 34 & 23 & 72 & \\
\hline Total & 100 & 100 & 100 & \\
\hline $\begin{array}{l}\text { Examen des anœstrus } \\
\text { du post-partum }\end{array}$ & 73 & 70 & 91 & $P<0,001$ \\
\hline Moyens de détection & & & & $P<0,001$ \\
\hline Détection visuelle & 81 & 59 & 53 & \\
\hline Crayon détecteur & 2 & 4 & 21 & \\
\hline Calendrier rotatif & 17 & 37 & 26 & \\
\hline Total & 100 & 100 & 100 & \\
\hline
\end{tabular}

des autres vaches (1 p. 100). La taille du troupeau ou sa spécialisation ont été sans influence sur la nature des signes pris en considération pour le diagnostic d'œstrus.

Dans 41 p. 100 des cas, le comportement astral des vaches était observé par les éleveurs en fonction de leur disponibilité. D'autres associaient cette activité de détection à la distribution d'aliments (39 p. 100) ou au rassemblement des animaux pour la traite (20 p. 100). L'observation des chaleurs a été significativement plus souvent associée à la distribution des aliments $(\mathrm{p}<0,01)$ dans les élevages de plus grande taille (tableau II) et dans les exploitations de type mixte $(\mathrm{p}<0,05)$ (tableau III). Dans la majorité des cas (76 p. 100), les éleveurs faisaient examiner par le vétérinaire les vaches en anœstrus du post-partum. Cette pratique était significativement $(\mathrm{p}<0,001)$ plus fréquente dans les élevages de plus de 60 femelles (tableau II). Elle n'était pas influencée par le type de spécialisation. Deux éleveurs sur trois (66 p. 100) n'utilisaient pas de moyens complémentaires de détection. Au nombre des moyens utilisés, on pouvait relever le calendrier rotatif (27 p. 100) et le crayon marqueur ( 7 p. 100). Ces moyens étant significativement $(\mathrm{p}<0,01)$ plus souvent utilisés dans les exploitations de plus grande taille (tableau II). De même, les élevages de type mixte utilisaient préférentiellement le crayon marqueur tandis que le calendrier rotatif était davantage utilisé dans les exploitations laitières $(\mathrm{p}<0,05)$ (tableau III). A la question de savoir quelle était la durée de la période d'attente observée, respectivement 20, 15, 33 et 32 p. 100 des éleveurs inséminaient pour la première fois leurs vaches avant 50 jours, entre 50 et 69 jours, entre 70 et 90 jours, et à plus de 90 jours après le vêlage. D'une manière générale mais non significative, la durée de la période d'attente a augmenté avec la taille de l'exploitation. Elle n'a pas été significativement différente dans les exploitations de type mixte ou laitier.

\section{Tableau III}

Effet du type de spécialisation sur la répartition en pourcentage des modalités pratiques de détection

\begin{tabular}{lrrr} 
& Mixte & Laitière & \multicolumn{1}{c}{ Test } \\
$\begin{array}{l}\text { Moments d'observation } \\
\begin{array}{l}\text { Pendant la distribution } \\
\text { d'aliment }\end{array}\end{array}$ & & $\mathrm{P}<0,05$ \\
Avant la traite & 54 & 30 & \\
Moment non spécifique & 35 & 25 & \\
Total & 100 & 100 & \\
Moyens de détection & & & $\mathrm{P}<0,05$ \\
$\quad$ Détection visuelle & 70 & 64 & \\
Crayon détecteur & 12 & 5 & \\
Calendrier rotatif & 18 & 31 & \\
Total & 100 & 100 &
\end{tabular}

\section{DISCUSSION}

L'enquête réalisée dans 222 exploitations bovines laitières ou mixtes a confirmé que la plupart (57 p. 100) des éleveurs avaient des problèmes de détection des chaleurs quelle qu'ait été la taille des exploitations concernées. La même observation a été faite par 42 p. 100 des éleveurs lors d'une enquête réalisée en France (26). Seegers et coll. (33) rapportent que la capacité de l'éleveur à détecter les chaleurs dépend de l'intensité et de la fréquence des signes comportementaux plus ou moins spécifiques manifestés par la vache. L'expression courte et discrète ainsi que le type de stabulation et même, pour certains auteurs, la taille importante des troupeaux peuvent donc rendre leur détection délicate et difficile. La présente enquête a montré que, par ordre d'importance, l'alimentation, le manque de temps, la race et le type de stabulation ont 
constitué, selon les éleveurs, les causes majeures d'une mauvaise détection. Le manque de temps a également été un facteur identifié par l'enquête française (14 p. 100 des éleveurs). Pour 61 p. 100 des éleveurs algériens, ce manque de détection aurait été imputable au manque de manifestation des chaleurs par l'animal. Cette possibilité n'est avancée que par 28 p. 100 des éleveurs français (33). La cause en serait $(8,23)$ un manque d'adéquation entre les apports énergétiques et le niveau de production laitière $(32,36)$. L'impact du nombre et du moment d'observation journalière sur la qualité de la détection est aussi réel $(4,27)$. Ainsi, van Vliet et van Eerdenburg (36) ont rapporté que deux observations journalières de 30 minutes à 12 heures d'intervalle s'accompagnaient d'un taux de détection de 70 p. 100 avec une précision de 100 p. 100 . De même, selon Rœlofs et coll. (27) le pourcentage de détection serait de 30 p. 100 lorsque le nombre d'observations est de trois par jours pendant 30 minutes. L'acceptation du chevauchement constitue le signe le plus caractéristique d'un état œstral $(7,35)$. Pourtant ce signe n'a été considéré comme caractéristique de l'œstrus que par 16 p. 100 des éleveurs, la majorité d'entre eux basant leur constat d'œstrus sur des signes mineurs dont on sait qu'ils sont sujets à de grandes variations individuelles (36).

L'enquête a révélé qu'un éleveur sur cinq inséminait ses vaches avant le cinquantième jour du post-partum et que deux éleveurs sur trois réalisaient cette première insémination plus de 70 jours après le vêlage. On peut y voir un effet indirect de la mauvaise qualité de la détection des chaleurs. Celle-ci contribue à augmenter l'intervalle entre le vêlage et la première insémination (période d'attente) et par conséquent l'intervalle entre vêlages $(16,17)$.

Il semble que, conscients de leur difficulté à observer les chaleurs et à les reconnaître, la majorité des éleveurs (76 p. 100) et surtout ceux qui géraient des troupeaux de plus de 60 femelles, faisaient examiner par un vétérinaire les vaches qui ne présentaient pas de chaleurs au cours des 50 premiers jours du post-partum (anœstrus). Cette pratique est essentielle pour distinguer les animaux cyclés mais dont les chaleurs ne sont pas détectées par l'éleveur (anœstrus dit de détection) de ceux qui présentent un anœstrus pathologique d'origine fonctionnelle, kystique ou pyométrale (11). Les éleveurs sont peu enclins (34 p. 100 dans la présente étude) à utiliser des systèmes d'aide à la détection. Ces moyens restent surtout utilisés dans les élevages comptant plus de 60 vaches laitières. Diverses études montrent pourtant que ces moyens augmentent la fréquence et l'exactitude du constat d'œstrus $(3,18,19)$.

\section{- CONCLUSION}

La détection des chaleurs demeure un problème majeur dans les élevages bovins algériens. Il faut y voir plusieurs raisons telles que le manque de formation des éleveurs à l'identification des signes caractéristiques de l'œstrus, leur insuffisante appropriation de cette importante pratique de conduite d'un cheptel reproducteur, la nature des stabulations, le nombre moyen de bovins par exploitation et le manque d'utilisation de moyens complémentaires de détection. Compte tenu de ses effets sur les performances de reproduction, la gestion de ce facteur d'élevage est essentielle. Certains éleveurs l'ont d'ailleurs bien compris puisqu'ils recourent au vétérinaire bien plus systématiquement que d'autres pour poser un diagnostic aussi précis que possible.

\section{Remerciements}

Les auteurs remercient le docteur J.-M. Beduin pour l'aide apportée à l'analyse statistique des résultats ainsi que tout le personnel du département des sciences vétérinaires de l'Université de Blida.

\section{BIBLIOGRAPHIE}

1. AT-TARAS E., SPAHR S.L., 2001. Detection and characterization of estrus in dairy cattle with an electronic heat mount detector and an electronic activity tag. An. Dairy. Sci. Assoc., 84: 792-798.

2. BOUZEBDA Z., BOUZEBDA F., GUELLATI M.A., GRAI N., 2006 . Evaluation des paramètres de la gestion de la reproduction dans un élevage bovin du nord est algérien. Sci. Tech. C., 24 : 13-16.

3. CAVALIERI J., FLINKER LR., ANDERSON G.A., MACMILLAN K.L., 2003. Characteristics of oestrus measured using visual observation and radiotelemetry. Anim. Reprod. Sci., 76: 1-12.

4. CAVESTANY D., FERNANDEZ M., PEREZ M., TORT G., SANCHEZ A., SIENA R., 2008. Oestrus behaviour in heifers and lactating dairy cows under a pasture-based production system. Vet. Quart., 30: 10-34.

5. COLLICK D.W., WARD W.R., DOBSON H., 1989. Associations between types of lameness and fertility. Vet. Rec., 125: 103-106.

6. DERIVAUX J., 1989. Reproduction chez les animaux domestiques. Liège, Belgique, Derouaux, 157 p. (T1), 175 p. (T2).

7. DRANSFIELD M.B.G., NEBEL R.L., PEARSON R.E., WARNICK L.D., 1998. Timing of insemination for dairy cows identified in estrus by a radiotelemetric estrus detection, system. J. Dairy Sci., 81: 1874-1882.

8. FERGUSON J.D., 2005. Nutrition and reproduction in dairy herds. Vet. clin. North Am. Food. Anim. Pract., 21: 325-347.

9. GALINA C.S., ARTHUR G.H., 1990. Review of cattle reproduction in the tropics. Part 4, Oestrous cycles. Anim. Breed., 58: 697-707.

10. GHORIBI L., BOUAZIZ O., TAHAR A., 2005. Etude de la fertilité et de la fécondité dans deux élevages bovins laitiers. Sci. Tech. C., 23 : 46-50.

11. HANZEN C., BASCON F., THERON L., LOPEZ-GATIUS F., 2008. Les kystes ovariens dans l'espèce bovine : Rappels physiologiques et étiopathogénie. Ann. Méd. Vét., 152 : 17-34.

12. HELMER S.D., BRITT J.H., 1985. Mounting behaviour as affected by stage of oestrous cycle in Holstein heifers. J. Dairy Sci., 68: 1290-1296.

13. HURNIK J.F., KING G.J., ROBERTSON H.A., 1975. Oestrus behaviour in post-partum Holstein cows. Appl. Anim. Ethol., 2: 55-58.

14. KERBRAT S., DISENHAUS C., 2004. A proposition for an updated behavioural characterization of the estrus period in dairy cows. Appl. Anim. Behav. Sci., 87: 223-238.

15. KILGOUR R., SKARSHOLT B.H., SMITH J.F., BREMNER K.J., MORRISON M.C.L., 1977. Observations on the behaviour and factors influencing the sexually active group in cattle. Proc. N. Z. Soc. Anim. Prod., 37: 128-135.

16. KING G.J., HURNIK J.F., ROBERTSON H.A., 1976. Ovarian function and estrus activity in dairy cows during early lactation. J. Dairy. Sci., 42: 688-692.

17. LARCETE G., 2003. La détection des chaleurs et le moment d'insémination. In : Symposium sur les bovins laitiers, Centre d'insémination artificielle de Québec, Saint-Hyacinthe, 30 oct. 2003.

18. LEHRER A.R., LEWIS G.S., AIZINBUD E., 1992. Estrus detection in cattle: recent developments. Anim. Reprod. Sci., 28: 355-361.

19. LEWIS G.S., AIZINBUD E., LEHER A.R., BROCKWAY B.P., 1988. A telemetry system for detecting dairy cows in estrus. J. Anim. Sci., 66 (suppl. 1).

20. LUCY M.C., 2003. Mechanisms linking nutrition and reproduction in postpartum cows. Soc. Reprod. Fertil., 61: 415-427.

21. MINISTERE DE L'AGRICULTURE ET DU DEVELOPPEMENT RURAL, 2007. Rapport annuel. Alger, Algérie, direction des Services statistiques.

22. MINISTERE DE L'AGRICULTURE ET DU DEVELOPPEMENT RURAL, 2009. Rapport annuel. Alger, Algérie, direction des Services statistiques.

23. ORIHUELA A., 2000. Some factors affecting the behavioural manifestation of oestrus in cattle. App. Anim. Behav. Sci., 70: 1-16.

24. PHILLIPS C.J.C., SCHOFIELD S.A., 1990. The effect of environment and stage of the estrus cycle on the behaviour of dairy cow. Appl. Animal. Behav. Sci., 27: 21-31.

25. PENNINGTON J.A., ALBRIGHT J.L., CALLAHAN C.J., 1986. Relationship of sexual activities in estrous cows to different frequencies of observation and pedometer measurements. J. Dairy Sci., 69: 2925-2934. 
26. PONSART C., FRAPPAT B., GATIEN J., CHANVALLON A., CONSTANT F., DISENHAUS C., SEEGERS H., BLANC F., RIBAUD D., SALVETTI P., PACCARD P., 2010. La détection par les éleveurs des chaleurs des vaches : des pratiques et des logiques de décision très diverses. Renc. Rech. Rum., 17.

27. ROELOFS J.B., VAN EERDENBURG F.J.C.M., SOEDE N.M., KEMP B., 2005. Various behavioral signs of estrous and their relationship with ovulation in dairy cattle. Theriogenology, 63: 1366-1377.

28. ROELOFS J.B., LOPEZ-GATIUS F., HUNTER R., VAN EERDENBURG F., HANZEN C., 2010. When is a cow in estrus? Clinical and practical aspects. Theriogenology, 74: 327-344.

29. ROELOFS J.B., VAN EERDENBURG F.J.C.M., SOEDE N.M., KEMP B., 2005. Pedometer readings for estrus detection and as predictor for time of ovulation in dairy cattle. Theriogenology, 64: 1690-1703.

30. RORIE R.W., BILBY T.R., LESTER T.D., 2002. Application of electronic estrus detection technologies to reproductive management of cattle. Theriogenology, 57: 137-148.

31. ROXSTROM A., STRANDBERG E., BERGLUND B., EMANUELSON U., PHILIPSSON J., 2001. Genetic and environmental correlations among female fertility traits, and between the ability to show oestrus and milk production in dairy cattle. Acta. Agric. Scand., 51: 192-199.

\section{Summary}

Yahimi A., Djellata N., Hanzen C., Kaidi R. Analysis of heat detection practices in dairy cattle farms in Algeria

A survey on heat detection practices was performed in 222 cattle farms spread over five regions (wilayas) of Central Algeria (Tizi Ouzou, Blida, Medea and Tipaza Bouira). The questionnaire contained general information on livestock (number of females, stabling, specialization) as well as heat detection practices (observation periods, signs observed) and complementary means as needed. The majority of farmers $(57 \%)$ faced a problem of heat detection. In $49 \%$ of the cases, they inseminated their cows for the first time from 50 to 90 days after calving. One farmer out of two (53\%) detected heats twice a day and $40 \%$ of them observed them three times a day, this activity being carried out at random. They primarily based their finding of estrus on the presence of a vulvar discharge $(19 \%)$ or on mounting acceptance $(16 \%)$. Herd size and specialization had no impact on the frequency of the signs considered. Cows that did not shown heat signs during the first 60 days after calving were clinically examined by a veterinarian in $76 \%$ of the farms. Additional means of detection such as rotating schedules or pencil marks were used only by $34 \%$ of the farmers. The factors responsible for heat manifestation, moments and numbers of observations, examination of post-partum anestrous, and use of additional means were influenced by the farm specialization and herd size, unlike heat signs, the waiting period and the difficulty of detection.

Keywords: Cattle - Dairy cow - Estrus detection Reproduction - Algeria.
32. SAKAGUCHI M., SASAMOTO Y., SUZUKI T., TAKAHASHIY., YAMADA Y., 2006. Fate of cystic ovarian follicles and the subsequent fertility of early postpartum cows. Vet. Rec.,159: 197-201.

33. SEEGERS H., BILLON D., BOSSARD-APPER E., PONSART C., PACCARD P., DISENHAUS C., GATIEN J., SALVETTI P., GRIMARD B., CHANVALLON A., BAREILLE N., 2010. Evaluation rétrospective de la qualité de la détection des chaleurs en troupeau bovin laitier à partir de données déjà disponibles. Rencontres Rech. Rumin., 17.

34. VAILES L.D., BRITT J.H., 1990. Influence of footing surface on mounting and other sexual behaviors of estral Holstein cows. J. Anim. Sci., 68: 2333-2339.

35. VAN EERDENBURG F.J.C.M., KARTHAUS D., TAVERNE M.A.M., MERICS I., SZENCI O., 2002. The relationship between estrous behavioral score and time of ovulation in dairy cattle. J. Dairy Sci., 85: 1150-1156.

36. VAN VLIET J.H., VAN EERDENBURG F.J.C.M., 1996. Sexual activities and œestrus detection in lactating Holstein cows. Appl. Anim. Behav. Sci., 50: 57-69.

37. XU Z.Z., MCKNIGHT D.J., VISHWANATH R., PITT C.J., BURTON L.J., 1998. Estrus detection using radiotelemetry or visual observation and tail painting for dairy cows on pasture. J. Dairy Sci., 81: 2890-2896.

Accepté le 31.05.2013

\section{Resumen}

Yahimi A., Djellata N., Hanzen C., Kaidi R. Análisis de las prácticas de detección de celos en los criaderos de bovinos de leche argelinos

Se realizó una encuesta sobre las prácticas de deteccion de celos en 222 establecimientos bovinos, repartidos en cinco regiones (wilayas) del centro de Argelia (Tizi Ouzou, Blida, Medea, Tipaza y Bouira). El cuestionario utilizado contenía informaciones generales, relativas al criadero (número de hembras, estabulación, especialización), así como también las prácticas de detección de calores (periodo de observación, signos observados) y los medios complementarios eventualmente utilizados. La mayoría de los criadores (57\%) estuvieron confrontados a un problema de detección de calores. Únicamente en $49 \%$ de los casos se inseminó a las vacas, por la primera vez, 50 a 90 días post parto. Uno de cada dos criadores (53\%) detectaba los celos dos veces por día y $40 \%$ los observaban tres veces por día, esta actividad se efectuó de manera aleatoria. La verificación del estro se basaba principalmente sobre la presencia de una descarga de la vulva (19\%) o sobre la aceptación de la monta (16\%). El tamaño del hato o la especialización no tuvieron influencia sobre la frecuencia de los signos considerados. Las vacas que no presentaron signos de calor durante los primeros 60 días post parto fueron objeto de un examen clínico por parte de un veterinario en $76 \%$ de los establecimientos. Los medios complementarios de detección, como el calendario rotativo o el lápiz detector fueron utilizados solamente por $34 \%$ de los criadores. Los factores responsables de la manifestación de los calores, los momentos y el número de observaciones, el examen de anestro post parto y la utilización de medios complementarios, fueron influenciados por la especialización y el tamaño del establecimiento, contrariamente a los signos de calor, el periodo de espera y la dificultad de detección.

Palabras clave: Ganado bovino - Vaca lechera - Detección del estro - Reproducción - Argelia. 
